

\section{Scripta Nova}

Revista Electrónica de Geografía y Ciencias Sociales Universitat de Barcelona

1 de Septiembre de 2020

\title{
LOS «BANCOS MALOS» Y LA RESOLUCIÓN DE LA CRISIS FINANCIERO-INMOBILIARIA EN EUROPA
}

\author{
Dr. Michael Byrne \\ School of Social Policy, Social Work and Social Justice, University College Dublin \\ michael.byrne@ucd.ie
}

Recibido: 25 enero 2019; Devuelto para correcciones: 27 agosto 2019; Aceptado: 28 enero 2020

\section{Los «bancos malos» y la resolución de la crisis financiero-inmobiliaria en Europa (Resumen)}

Este artículo se centra en las Sociedades de Gestión de Activos (SGA), o «bancos malos», creadas por parte de los gobiernos para adquirir y gestionar activos improductivos, a menudo vinculados con el sector inmobiliario, tras las crisis bancarias sistémicas. [Este artículo examina ciertas SGA que se han creado dentro de Europa como parte de las respuestas de estados miembros e instituciones europeas a la crisis bancaria. El artículo analiza las SGA desde cuatro perspectivas para comprender sus dimensiones específicamente urbanas. En primer lugar, las SGA siguen con la extracción financiarizada de rentas del espacio urbano. En segundo lugar, actúan como «creadores de mercados» al devolver «liquidez» al sector inmobiliario. En tercer lugar, contribuyen a la globalización del sector inmobiliario al intensificar los circuitos que vinculan los mercados inmobiliarios locales con flujos globales de capital. Por último, generan contestación política en torno al acceso a y el control sobre el espacio urbano.

Palabras clave: financiarización, Sociedades de Gestión de Activos, bancos malos, crisis financiera, activos tóxicos, economía política urbana.

\section{The «bad banks" and the resolution of the financial-real state crisis in Europe (Abstract)}

This article focuses on Asset Management Companies (AMCs), or 'bad banks', which are established by governments to acquire and manage non-performing assets, often linked to real estate, in the wake of systemic banking crises. The article looks at AMCs which have been established within Europe as part of member state and European institution responses to the baking crisis. The article analyses AMCs through four lenses to capture their distinctly urban dimensions. Firstly AMCs continue the financialized extraction of value from urban space. Secondly, they act as 'market makers' by restoring the 'liquidity' of real estate. Thirdly they contribute to the globalization of real estate by intensifying the circuits linking local real estate with global pools of capital. Fourthly they generate political contestation around access to and control of urban space.

Key words: financialization, Asset Managanement Companies, bad Banks, financial crisis, toxic assets, urban political economy 
Tras la Crisis Financiera Global (CFG), los investigadores siguen examinando la creciente interdependencia entre las finanzas y el espacio urbano. ${ }^{1}$ Aunque hay diferentes perspectivas sobre este proceso, la mayoría coincide en que el sector inmobiliario, y, por ende, el espacio urbano, ha sido y sigue siendo clave en la expansión del sistema financiero. También existe consenso en que la integración del sector inmobiliario y las finanzas es algo inherentemente propenso a las crisis, con la CFG en sí sirviendo de ejemplo paradigmático. A pesar de este hincapié en las crisis, hay muy pocos trabajos sobre cómo se solucionan las crisis financieras e inmobiliarias. ¿Cómo se vuelve a encarrilar la financiarización de la ciudad tras las sistemáticas y cíclicas crisis a las que es tan propensa? ¿Y qué papel juega el estado, no solo en posibilitar las burbujas, sino también en resolver las crisis?

Este artículo se centra en un tipo de institución en concreto que ha jugado un papel clave en la resolución de la CFG en el contexto europeo: las Sociedades de Gestión de Activos (SGA) o «bancos malos». Dos de las entidades con más activos inmobiliarios en Europa desde 2008 han sido Sociedades de Gestión de Activos: la National Asset Management Agency (Agencia Nacional de Gestión de Activos: NAMA) y la Sociedad de Gestión de Activos Procedentes de la Reestructuración Bancaria (SAREB). Éstas fueron creadas por los gobiernos de Irlanda y España, respectivamente, para adquirir deuda inmobiliaria "tóxica» tras la crisis financiera e inmobiliaria que estalló en ambos países en 2008. En 2014, las SGA representaron aproximadamente el $53 \%$ de las ventas de activos inmobiliarios comerciales en Europa ${ }^{2}$. A nivel mundial, se han creado SGA tras las crisis financiero-inmobiliarias y son una forma clave de intervención gubernamental para lidiar con las crisis bancarias sistémicas. Ayudan a devolver la solvencia a los bancos, pero también actúan como una suerte de almacén para activos inmobiliarios tóxicos, además de ser un mecanismo para resolver los préstamos morosos y los activos deteriorados. Y aun así, a pesar de las enormes cantidades de bienes inmuebles que controlan y el papel clave que juegan en reactivar mercados, hay poca literatura que trate las implicaciones urbanas de las SGA.

Este artículo responde a esta falta mediante el estudio de la economía política urbana de las SGA. Proporciona un resumen del papel de las SGA en responder a las crisis bancarias y en dar solución a los activos inmobiliarios deteriorados o "tóxicos». También demuestra que aunque las SGA constituyen, en principio, una intervención en el sector bancario, tienen impactos decisivos sobre las ciudades debido a la integración del sistema financiero y los activos inmobiliarios. Con especial referencia a ejemplos de SGA en España y Irlanda, pero nutriéndose de la experiencia internacional en general, el artículo propone que las intervenciones estatales en el sistema financiero son un importante objeto de estudio para investigadores urbanos y aquellos que están interesados en la dimensión urbana de la financiarización.

1 Me gustaría agradecer a Jean Duggan por la traducción de este artículo y a Dr. Mauro Castro por su ayuda en la preparación de lo mismo. Este trabajo ha sido financiado por la beca de investigación «Government of Ireland Postdoctoral Fellowship» del Irish Research Council. 2 Cushman \& Wakefield, 2014, p. 3 
Además, las SGA han jugado un papel especialmente importante en la manera en que los estados miembros y las instituciones de la Unión Europea han intentado resolver las crisis sistémicas bancarias que proliferaron en todo el continente tras la crisis financiera.

La principal conclusión del artículo es que la dimensión urbana de las SGA puede ser conceptualizada desde cuatro perspectivas. En primer lugar, las SGA suponen la extracción de rentas del espacio urbano y su apropiación por actores financieros. En segundo lugar, las SGA devuelven la liquidez a los activos inmobiliarios en tiempos de crisis y así «crean el mercado». En tercer lugar, la devolución de la liquidez y la «creación de mercados» pueden suponer una intensificación del vínculo entre el mercado inmobiliario local y los flujos globales de capital. En cuarto lugar, el hecho de que las SGA están situadas en la intersección entre el estado, las finanzas y el sector inmobiliario significa que generan nuevas controversias en torno al acceso a y el control sobre los espacios urbanos devaluados. Además, examinar cómo la resolución de las crisis bancarias sistémicas está inherentemente vinculada a las dimensiones urbanas de las SGA arroja luz sobre la co-constitución del espacio urbano y la financiarización.

Lo que se expone a continuación está basado principalmente en datos de dos SGA públicas destacadas: la NAMA y la SAREB. He llevado a cabo una investigación empírica a fondo sobre la NAMA, incluyendo entrevistas extraoficiales con personal de la NAMA y un alto cargo del gobierno durante el período de su creación, además de una considerable investigación documental utilizando informes y material publicado por el Department of Finance, que tiene competencia para supervisar el «banco malo» irlandés. Los datos relativos a la SAREB provienen también de documentos públicamente disponibles. Para situar los ejemplos de Irlanda y España en la experiencia internacional más general, recurro a estudios sobre la Resolution Trust Corporation (RTC) en EEUU, que se creó como respuesta a la crisis de «ahorros y préstamos» de principios de los años noventa 3 . Existe un conjunto de literatura secundaria sobre la RTC que es principalmente descriptiva, pero también hay varias intervenciones analíticas y teóricas significativas que indagan en sus implicaciones con respecto a los estudios urbanos. Esto se ve complementado con referencias a la literatura económica disponible, publicada principalmente por instituciones internacionales (por ejemplo, el FMI y el Banco Mundial) y empresas internacionales de asesoría inmobiliaria y financiera.

En el primer apartado, el artículo hace un repaso a la literatura sobre las dimensiones urbanas de la financiarización. A continuación, proporciona una introducción y un resumen global de las SGA y cómo éstas responden a las crisis bancarias. El tercer apartado del artículo analiza las dimensiones urbanas de las SGA siguiendo las cuatro líneas mencionadas. El cuarto utiliza este análisis para teorizar más a fondo sobre las SGA y su importancia desde el punto de vista de las dimensiones urbanas de la financiarización. El artículo proporciona pruebas sólidas de que las SGA

3 Gotham, 2006 
operan como aparatos concretos para reanimar y reactivar la «financiarización de la ciudad» tras las crisis sistémicas, y permite una mejor comprensión de la relación entre la financiarización y el espacio urbano.

\section{El espacio urbano y el proceso de financiarización}

El concepto de financiarización se refiere al creciente poder e influencia de procesos y actores financieros a nivel social, económico y político ${ }^{4}$. Con respecto al espacio urbano, este creciente poder ha sido conceptualizado principalmente desde el punto de vista de tres procesos interrelacionados: el importante incremento de la "liquidez» de los inmuebles, la transformación de los inmuebles en activos cuasi-financieros y la creciente disponibilidad de capital para la inversión inmobiliaria ${ }^{5}$. Los bienes inmuebles son tradicionalmente un activo ilíquido cuando se los compara, por ejemplo, con las acciones y las participaciones, como resultado del alto grado de territorialización y los rasgos «únicos» del lugar ${ }^{6}$. Esto es lo que Gotham ${ }^{7}$ llama el problema de la «fijeza del espacio» (spatial fixity).

Sin embargo, la financiarización del mercado inmobiliario ha tenido un impacto dramático en cuanto a la superación de los límites a la acumulación asociados al lugar como mercancía ${ }^{8}$. Esto ha tenido lugar mediante varias formas de innovación financiera, incluyendo el proceso de titulización o la aparición de las sociedades de inversión inmobiliaria (real estate investment trusts) ${ }^{9}$. Tales innovaciones operan para extraer flujos de ingresos (es decir, reembolsos de deuda o ingresos por alquiler) de los inmuebles de los que provienen. La creciente liquidez de los inmuebles está vinculada a su transformación en activos financieros ${ }^{10}$. Las inversiones en algunos tipos de inmuebles ahora se pueden negociar fácilmente en mercados globales, lo que permite a los inversores ver tales inversiones como «activos rentables negociables» (tradable income-yielding assets) ${ }^{11}$. Como un informante del sector privado me comentó, los bienes inmuebles se sitúan «convenientemente entre las acciones y los bonos en el sentido de que producen un flujo fijo de ingresos (como los bonos) mientras a la vez ofrecen posibilidades para la revalorización del capital (como las acciones). Además, mientras el mercado inmobiliario se ha «financiarizado», el sistema financiero en sí ha pasado por una transformación importante y una expansión dramática como resultado de la desregulación, la liberalización de los mercados de capitales y el cambio de divisas, y un período prolongado de tipos de interés históricamente bajos en economías claves ${ }^{12}$.

\footnotetext{
4 Blackburn, 2006; Kelly, 2014

5 Gotham, 2009; Fields, 2014; Moreno, 2014; Harvey, 2006

6 Carruthers \& Stinchcombe, 1999; Weber, 2002; Gotham, 2006; Christophers, 2010

7 Gotham, 2009, p. 357

8 Rutland, 2010; Christophers, 2010

9 Gotham, 2006; Rutland, 2010

10 Christophers, 2010

11 Guironnet \& Halbert, 2014; Coakley, 1994

12 Brenner, 2006; López \& Rodríguez, 2010; 2011; Lapavitsas, 2013
} 
Los investigadores también han destacado el papel clave que juega el estado en estos procesos. En particular, la reducida oferta de viviendas sociales, la desregulación de los mercados de alquiler ${ }^{13}$ y la merma de la financiación del gobierno local por parte del gobierno central, llevando a la dependencia del primero en el capital privado $^{14}$. Los aspectos adicionales abarcan todo, desde la normativa que posibilita las sociedades de inversión inmobiliaria a entornos fiscales que favorecen las finanzas especulativas ${ }^{15}$.

En conjunto, estas dinámicas se pueden conceptualizar desde el punto de vista de la financiarización del espacio urbano ${ }^{16}$, un proceso que es especialmente visible en sus efectos negativos sobre el entorno construido y la inestabilidad económica. La crisis financiera global ha dejado esto sobradamente claro, mostrando como el impago por parte de propietarios de viviendas con bajos ingresos en algunos estados de los EEUU ha desencadenado el caos económico global, dando lugar a su vez a una ola de embargos ${ }^{17}$, crisis habitacionales en muchos países y entornos urbanos obsoletos $^{18}$. Es importante destacar aquí que, según los estudios, la financiarización de la ciudad intensifica las dinámicas cíclicas de auge y caída que desde hace tiempo caracterizan el mercado inmobiliario ${ }^{19}$.

La tendencia hacia las crisis también aparece en otras investigaciones centradas en estos temas desde el punto de vista de la transformación de la acumulación de capital. Estos trabajos tienden a entender el creciente poder de las finanzas como una respuesta a la crisis manufacturera o del capitalismo industrial asociado a los años setenta ${ }^{20}$. Examinan, desde distintas perspectivas, como el espacio urbano y el entorno construido pueden servir para gestionar las contradicciones del proceso de acumulación. Sin duda, el trabajo de David Harvey ${ }^{21}$ es la aportación más importante en este sentido. Sostiene que en respuesta a las crisis de sobreacumulación en el terreno de la producción capitalista, el capital puede huir al entorno construido. Las entidades financieras juegan un papel clave en esto, pues proporcionan el canal mediante el cual el capital se dirige hacia la producción de espacios urbanos y también los mecanismos para capturar la riqueza que proviene de esos espacios ${ }^{22}$. En este proceso, sin embargo, la crisis de la acumulación de capital no se supera, sino que se desplaza al entorno construido. La experiencia reciente de Europa está fundamentalmente vinculada a la experiencia de la Unión Económica y Monetaria (es decir, la formación del euro). La UEM dio lugar a la desregulación financiera, la eliminación del riesgo de tipo de cambio, persistentes tipos de interés bajos fijados por el Banco

13 Fields, 2014; McCabe, 2011

14 Raco \& Henderson, 2009; Guironnet \& Halbert, 2014

15 Gotham, 2006; McCabe, 2011; MacLaran \& Kelly, 2014

16 Moreno, 2014

17 Colau \& AleMAny, 2014

18 O'Callaghan et al., 2014

19 Fields, 2014; López \& Rodríguez, 2010

20 van der Zwan, 2012

21 Harvey ,2006, 2012

22 Moreno, 2014 
Central Europeo y un consecuente incremento dramático en los flujos de capital transfronterizos. En particular, estados miembros periféricos como Irlanda y España consiguieron acceso a una verdadera oleada de crédito barato, permitiendo una prolongada burbuja crediticia e inmobiliaria que se mantuvo durante gran parte de los $2000^{23}$.

El papel del estado y la cuestión de las crisis cíclicas son especialmente relevantes en el período pos-crisis. En los últimos años, los investigadores han identificado una variedad de procesos a través de los cuales la financiarización de la vivienda y el espacio urbano ha sido rearticulada ${ }^{24}$. Los activos deteriorados o "tóxicos» han tenido un papel importante en este sentido ya que han sido reciclados por nuevos actores financieros para crear nuevas formas de financiarización de la vivienda ${ }^{25}$. Se ha prestado mucha atención al papel de los inversores institucionales en el rápido crecimiento de la vivienda en alquiler en muchos territorios, por ejemplo, EEUU ${ }^{26}$, Alemania ${ }^{27}$ e Irlanda ${ }^{28}$. Fields ${ }^{29}$ argumenta que «la financiarización se está extendiendo a nuevos terrenos, y nuevos modos de extracción de rentas financieras están surgiendo». En España, las sociedades de inversión inmobiliaria y las empresas de capital inversión estadounidenses (como Blackstone) han emprendido una ola masiva de inversión a través de la adquisición de bloques de pisos considerablemente desvalorizados después del $2008^{30}$, mientras en los EEUU, las empresas de capital inversión se han convertido en los mayores arrendadores del país a través de la adquisición de viviendas unifamiliares ejecutadas, una forma de propiedad residencial previamente poco común en el sector del alquiler ${ }^{31}$. Esta literatura, por ende, se enfoca en dinámicas macroeconómicas, particularmente en la necesidad del sistema financiero de encontrar avenidas para nuevas rondas de inversión especuladora.

De especial interés para los fines de este artículo, sin embargo, es la cuestión del papel que tiene el estado en la transición desde un ciclo de financiarización a otro. Aquí, los bancos malos han tenido un papel crucial, aunque poco explorado. En muchos territorios, como se analiza más abajo, se han convertido en un componente crucial de la intervención estatal en los sistemas financieros tras la crisis, particularmente en lo que respecta a los activos deteriorados. En hacer esto, actúan como un tipo de interfaz entre los activos tóxicos generados por la Crisis Financiera Global y el reciclaje de esos activos en nuevas olas de financiarización. Esto, junto con su naturaleza como entidades públicas y su gran escala, hace que estas instituciones sean de especial interés para los investigadores de la financiarización.

23 Norris \& Byrne, 2014

24 Waldron, 2018; Beswick et al., 2016

25 Beswick et al., 2016

26 Fields, 2018; Immergluck and Law, 2014

27 Wijburg and Aalbers, 2017; Kitzmann, 2017

28 Byrne, 2019

29 Fields, 2018, p.119

30 Beswick et al., 2016

31 Fields, 2018 


\section{Los bancos malos y las crisis financieras}

Tal y como se ha señalado, las SGA se crean cuando la viabilidad de un único banco o del sector bancario en su conjunto se encuentra amenazada por la proliferación de «activos deteriorados». El volumen de estos activos normalmente sube de forma dramática durante las crisis financieras ${ }^{32}$ cuando el número de deudores en dificultades o morosos incrementa y el valor de las garantías subyacentes se desploma. Todas las categorías de activos son susceptibles a este peligro, pero los cambios en los precios de los activos inmobiliarios son particularmente cíclicos y como tal están entre los activos deteriorados más comunes ${ }^{33}$. Esto puede debilitar la capitalización y solvencia de las entidades financieras, introducir incertidumbre generalizada en relación con el valor de los activos e impedir la vuelta a la concesión de crédito, amenazando la viabilidad del sistema financiero ${ }^{34}$. Así, la literatura económica mantiene que "la correcta gestión y enajenación de activos deteriorados es una de las tareas más críticas y complejas de una reestructuración bancaria exitosa y rápida ${ }^{35}$. Por eso, las respuestas estatales a las crisis financieras muchas veces suponen: (a) responder a la incertidumbre sobre el valor y el alcance de los activos deteriorados en manos de las entidades financieras; (b) "cristalizar» las pérdidas asociadas a los activos deteriorados; y (c) gestionar/reestructurar los activos deteriorados. Las SGA son una de las formas más comunes en las que los gobiernos llevan a cabo estas tareas $^{36}$. Como dicen los asesores internacionales McKinsey ${ }^{37}$, se han convertido en «una de las formas preferidas de despejar la duda y encontrar un nuevo comienzo». Los bancos malos lo consiguen al adquirir activos deteriorados directamente de una o más entidades financieras, y así, en efecto, «limpian» los balances de los bancos. Las SGA se han creado tras muchas de las crisis financieras e inmobiliarias que han tenido lugar en las últimas décadas, incluyendo la crisis de ahorros y préstamos de EEUU a finales de los años ochenta y principios de los años noventa, la crisis bancaria nórdica del mismo período y las crisis financieras asiáticas de finales de los años noventa y en la actualidad.

Una vez terminada la fase de adquisición de activos, el objetivo de las SGA es maximizar el valor de ellos. Por tanto, tienen un carácter fundamentalmente comercial en cuanto a su enfoque de gestión y enajenación de activos ${ }^{38}$. Esto supone o la venta rápida y directa de activos o cierto grado de reestructuración "para hacer que los activos sean económicamente viables y, así, atractivos para el comprador ${ }^{39}$. Aunque las SGA obtienen ingresos de una variedad de fuentes, como los que provienen de los alquileres de propiedades ejecutadas, operan en un período de tiem-

32 Claessens et al., 2011

33 Reinhart \& Rogoff, 2009; Claesens et al., 2011; Kilngebiel, 2000

34 Bacon, 2009

35 Klingebiel, 2000, p. 4

36 Claesens et al., 2011; Klingebiel, 2000; Stutts \& Watts, 2009; Ingves et al., 2004; Fung et al., 2004

37 McKinsey, 2012, p. 1

38 Klingebiel, 2000; Ingves et al., 2004; Fung et al., 2004

39 Klingebiel, 2000, 7; véase también Fung et al., 2004 
po relativamente corto, y su objetivo general es la enajenación de todos los activos mediante la venta. Esto puede ocurrir a través de la venta directa o la creación de «carteras» u otros mecanismos para juntar activos en paquetes más grandes ${ }^{40}$.

Tabla 1. SGA creadas para dar respuesta a la crisis financiera en países europeos

\begin{tabular}{llcc}
\hline Nombre & País & Fundada en & $\begin{array}{c}\text { Total de los activos } \\
\text { originales (en euros) }\end{array}$ \\
\hline NAMA & Irlanda & 2009 & 73,4 mil millones \\
EAA & Alemania & 2009 & 15,9 mil millones \\
KA Finanz & Austria & 2009 & 3,6 mil millones \\
FMS & Alemania & 2010 & 19,7 mil millones \\
UKAR & Reino Unido & 2010 & 107 mil millones \\
IBRC & Irlanda & 2011 & 21,9 mil millones \\
SAREB & España & 2012 & 107 mil millones \\
Propertize & Países Bajos & 2013 & 7,4 mil millones \\
DUTB & Eslovenia & 2013 & 1,3 mil millones \\
BES bad bank & Portugal & 2014 & 2,9 mil millones \\
Finansiel Stabilitet & Dinamarca & 2008 & 3,1 mil millones \\
HETA & Austria & 2014 & 1,6 mil millones \\
\hline
\end{tabular}

Este artículo se centra en las SGA establecidas tras la crisis financiera dentro de la Unión Europea (véase Tabla 1). Se han establecido SGA en más de doce países europeos y han sido una parte importante de la respuesta general de Europa a la crisis bancaria ${ }^{41}$. Aquí me centro en particular en los dos casos más significativos: la SAREB en España y la NAMA en Irlanda, ambas vinculadas a los Memorándums de Entendimiento acordados con la «Troika» como parte de los planes de rescate de Europa y el FMI. Junto con la liquidez de emergencia, la recapitalización y la nacionalización parcial o total, las SGA son una de las formas más importantes de intervención estatal asociadas a la gestión de las crisis en Europa. Sin embargo, también me refiero aquí a la Resolution Trust Corporation (RTC) para aportar mayor contexto y conocimientos adicionales ${ }^{42}$. Esta institución tuvo influencia tanto en el caso de la NAMA como el de la SAREB. Aunque las SGA adquieren grandes volúmenes de bienes inmuebles, a estos activos se les trata como activos fundamentalmente financieros, sin mucha preocupación por sus características espaciales o su extensión

40 Byrne, 2016

41 Gutiérrez \& Domènech, 2017

42 El RTC es una SGA que operó entre 1989 y 1995 como respuesta a la llamada «savings and loans crisis» de aquellos años. 
geográfica. Por ende, no es fácil encontrar información sobre la ubicación y naturaleza de los activos. Sin embargo, algunas observaciones sobre los casos de Irlanda y España pueden arrojar luz sobre esto. La NAMA únicamente adquirió préstamos vinculados a inmuebles (es decir, no adquirió ningún activo inmobiliario directo). Tras completar la adquisición, la cartera de préstamos de la NAMA quedó garantizada por más de 60.000 unidades inmobiliarias con posibilidades de venta ${ }^{43}$. Los tipos de activos se desglosan más o menos de la siguiente manera: oficinas ( $16 \%$ ); promoción ( $23 \%)$; suelo ( $11 \%)$; locales comerciales $(20 \%)$; hoteles y ocio $(9 \%)$; industrial (3\%); residencial ( $12 \%)$; otros $(5 \%)^{44}$. El $67 \%$ de las propiedades irlandesas están ubicadas en Dublín, con el $94 \%$ concentrado en los principales núcleos urbanos. La alta concentración de activos en zonas urbanas refleja el hecho de que las ciudades irlandesas han sido el foco del desarrollo especulativo, particularmente con respecto a la propiedad comercial, y que por tanto estas zonas presentaron altas concentraciones de activos tóxicos tras el estallido de la crisis.

La SAREB adquirió casi 200.000 activos valorados en algo más de 50 mil millones de euros. Aunque, como la NAMA, la SAREB se centra exclusivamente en activos inmobiliarios, la naturaleza de los activos adquiridos es un tanto diferente. En primer lugar, la SAREB adquirió tanto activos financieros (principalmente préstamos a empresas promotoras) como activos inmobiliarios directos. El 80\% eran activos financieros y el $20 \%$ restante eran activos inmobiliarios directos ejecutados por los bancos participantes. Asimismo, la SAREB adquirió una gran cartera de viviendas y activos financieros garantizados con viviendas ${ }^{45}$. Los tipos de activos inmobiliarios directos de la SAREB se desglosan de la siguiente manera: $56 \%$ residencial; $26 \%$ inmuebles complementarios; $11 \%$ suelo; $5 \%$ propiedad comercial (locales comerciales, propiedad industrial, oficinas y hoteles). El desglose de activos financieros por garantía subyacente es el siguiente: $41 \%$ residencial; $28 \%$ suelo; $10 \%$ locales comerciales, propiedad industrial y oficinas; $10 \%$ otras garantías; $11 \%$ sin garantías.

A continuación, exploro en mayor detalle cómo esto ocurre para poder desarrollar un análisis de las dimensiones urbanas de las SGA y el papel del espacio urbano en la resolución de las crisis financieras. Estos temas se examinan bajo los siguientes epígrafes: la extracción de valor del espacio urbano; liquidez y creación de mercados; la globalización del sector inmobiliario; y la impugnación contra los bancos malos.

\section{La dimensión urbana de las SGA La extracción de rentas del espacio urbano}

Las SGA siguen con el proceso de extraer rentas del espacio urbano para el sistema financiero. Al nivel más básico, a las SGA se les asignan la tarea de maximizar el valor comercial de los activos y, por ende, la de tratar los bienes inmuebles como

43 Department of Finance, 2014

44 Véase http://www.nama.ie/about-our-work/key-figures/

45 Gutiérrez \& Domènech, 2017 
activos financieros ${ }^{46}$. En la práctica, esto puede adoptar varias formas, desde recibir «pasivamente» flujos de ingresos que provienen de los reembolsos de deuda a estrategias más activas de reestructuración de la deuda o la toma de control de la garantía subyacente. Al hacer esto, las SGA pueden ser más agresivas con sus acreedores. Un informe de McKinsey ${ }^{47}$ sobre los «bancos malos» establecidos tras la CFG destaca que «dado que no tiene incentivo para mantener la relación con el cliente para futuros negocios, el banco malo puede ser mucho más firme en sus prácticas de resolución.» El mismo informe propone que las SGA auspiciadas por el gobierno, el tipo que consideramos aquí, pueden usar su conexión con el gobierno para hacer que los clientes sean más obedientes:

Los prestatarios, con razón, buscarán evitar un conflicto con el gobierno. Durante el proceso de venta de carteras, un intermediario que llevaba a cabo la venta de una cartera para un banco malo utilizó esta táctica con éxito y convenció a los prestatarios (que tenían otras relaciones con el gobierno) de abandonar su oposición a la venta de su préstamo ${ }^{48}$.

En términos más generales, una motivación clave para crear una SGA es que pueden seguir estrategias de maximización de rentas más intensivas y centradas que los bancos atribulados de quienes adquieren los activos ${ }^{49}$.

En muchos casos, la reproducción del trato del espacio urbano como activo financiero se agrava aún más por la relación continua entre las SGA y el sector bancario. Como se ha señalado anteriormente, las SGA a menudo emiten valores con garantía del estado (es decir, bonos) a los bancos de quienes adquieren activos como una recompensa por esos activos ${ }^{50}$. Los bancos se liberan de los activos tóxicos a cambio de bonos garantizados por el estado, un activo valioso de bajo riesgo, en lo que Joseph Stiglitz ${ }^{51}$ llama un cambio de "dinero por basura" (cash for trash). Este es el caso actual de la SAREB ${ }^{52}$ y la NAMA ${ }^{53}$. En estos casos, las SGA utilizan los ingresos generados mediante la venta de activos para pagar los bonos que emiten. En este sentido, los bancos obtienen los ingresos que provienen de los activos sin tener que mantener los activos en sus libros ni pagar por el coste de gestionarlos y las SGA disfrutan de unas facultades legales y operativas extraordinarias que les permite extraer rentas más efectivamente. La NAMA, por ejemplo, tiene el principal objetivo de gestionar «activos para optimizar, y capturar para fines de servicio de la deuda, su potencial para generar ingresos ${ }^{54}$ » (énfasis propio). En este sentido, Peter Bacon, autor de un informe gubernamental que llevó al establecimiento de la NAMA, describió a la sociedad como un tipo de "agencia de cobros" turbo. De manera similar, el

\footnotetext{
46 Byrne, 2016

47 McKinsey, 2012, p. 9

48 McKinsey, 2012, p. 10

49 Bacon, 2009

50 O'Broin, 2012

51 Stiglitz, 2009

52 Gutiérrez \& Domènech, 2017

53 Byrne, 2016

54 NAMA, 2012, p. 43,
} 
principal objetivo de la SAREB es «vender activos en búsqueda de niveles máximos de beneficio ${ }^{55}$.

Por lo tanto, las SGA operan en cierto sentido como un aparato establecido por el estado para consolidar y fortalecer la extracción de rentas del sector inmobiliario y encauzarlo hacia el sistema financiero, específicamente hacia los mismos bancos culpables de la concesión irresponsable de préstamos. Además, las SGA asumen el riesgo asociado a los activos, lo cual puede, a su vez, alimentar la crisis de deuda soberana $^{56}$.

\section{Liquidez y creación de mercados}

Las SGA, sin embargo, tienen una función más amplia en cuanto a la relación entre las finanzas y el espacio urbano, dado que su intervención abarca los mercados inmobiliarios financiarizados en general. Aquí se trata de cómo las SGA responden a una crisis de iliquidez generalizada en el mercado. Igual que la integración de los inmuebles y las finanzas puede intensificar la liquidez del primero, en períodos de crisis los activos inmobiliarios financiarizados pueden volverse ilíquidos ${ }^{57}$. Durante este tipo de crisis, determinar el valor de activos inmobiliarios deteriorados conlleva dificultades. Cuando los activos se deprecian de forma rápida, los mercados típicamente se paralizan y puede haber un número severamente reducido de transacciones en relación con un determinado conjunto de activos. Sin transacciones frecuentes, el precio y el valor son difíciles de determinar. Los bienes inmuebles, desde luego, también están asaltados por las características tradicionales de la iliquidez ya planteadas. Los activos (ej. activos inmobiliarios directos, además de préstamos y productos derivados) están respaldados por edificios específicos, y cada edificio es una mercancía más o menos única y muy influenciada por factores locales que pueden ser de difícil determinación para los potenciales compradores ${ }^{58}$. Los mercados de deuda también son un factor aquí. El sector inmobiliario es típicamente una industria local y la compra de bienes inmuebles se financia normalmente a través de deuda emitida por entidades bancarias locales. ${ }^{59}$ Una crisis inmobiliaria puede llevar a la quiebra generalizada de inversores inmobiliarios y puede dar lugar a problemas de solvencia a lo largo del sistema bancario local; - de hecho, la función de las SGA es responder a esta última situación. Así, puede que no haya crédito disponible y que no existan inversores potenciales. El problema de la iliquidez, entonces, se expresa en tres factores interrelacionados: los precios son difíciles de determinar; los compradores son difíciles de encontrar; y no hay financiación disponible.

Aunque su función principal es lidiar con las crisis bancarias, las SGA también deben vender sus activos al mejor precio posible. Donde no exista mercado, pueden

55 SAREB citado en García-Lamarca, 2017

56 O'Broin, 2012

57 Coakley, 1994

58 Gotham, 2006, 2009; Carruthers \& Stinchcome, 1999

59 Aunque es importante destacar que esto está cambiando como resultado de la financiarización del sector inmobiliario (Wissoker et al., 2014). 
tratar de crear uno para cumplir con este objetivo ${ }^{60}$. Esto lo hacen de varias maneras. Para empezar, cuando las SGA adquieren activos, tienen que determinar el precio que pagarán. En muchos casos, pagan el valor «contable» o «nominal» (ej. Securum en Suecia). En otros casos, pagan el valor actual de mercado o algo parecido a él (ej. la NAMA y la SAREB). Esto supone un amplio proceso de valoración, que puede dar al mercado un indicio del valor real de los activos, pero también en general ayuda a «fijarle un suelo a los precios inmobiliarios» ${ }^{61}$. Tras la adquisición de activos, y con mayor importancia, las SGA pueden limitar la oferta para «evitar abrumar los mercados locales de préstamos/activos con ventas "a precio de saldo"» ${ }^{62}$, y así, en efecto, moldean los mercados ${ }^{63}$. Limitar la oferta es particularmente importante porque los bancos probablemente intenten vender con urgencia los activos susceptibles a un deterioro dramático en su valor, en parte motivados por los requerimientos de capital, mientras las SGA pueden alejarse de tales preocupaciones y actuar en el interés del mercado en su conjunto. En ese sentido, operan efectivamente como un instrumento de política económica del gobierno para reforzar los mercados inmobiliarios financiarizados. Hay muchos ejemplos de intervenciones de este tipo, como las SGA nórdicas como el Securum sueco ${ }^{64}$, la RTC en EEUU ${ }^{65}$ y ejemplos actuales como la NAMA $^{66}$.

Al establecer los precios y controlar la oferta, las SGA pueden actuar como "creadores de mercados». Pero también lo hacen mediante el estímulo directo de transacciones, principalmente a través de las "estrategias de gestión de activos». En muchos casos, las SGA proporcionan "financiación de las ventas»; es decir, prestan dinero directamente a los compradores de sus activos. Dada la típica falta de financiación durante las crisis, esto da un impulso importante al mercado. Además, las SGA, incluyendo la RTC, la NAMA y la SAREB, pueden crear Empresas Conjuntas con los compradores de sus activos, en las cuales el «banco malo» en cuestión conserva una participación en el activo o el conjunto de activos. De este modo, sigue expuesto al riesgo asociado al activo, lo cual ayuda a atraer a compradores en un contexto de incertidumbre. Otras formas de gestión de activos incluyen la creación de carteras, donde las SGA juntan activos en grandes paquetes que diversifican el riesgo y consiguen una escala atractiva para las grandes entidades financieras (analizado más abajo). Esto puede suponer la creación de carteras específicamente confeccionadas para las estrategias de negocio de una empresa en concreto ${ }^{67}$. Por último, las SGA a veces crean productos financieros o formas de ingeniería financiera completamente nuevos con el fin de devolver la liquidez a cierto activo o conjunto de activos y al

60 Kitchin et al., 2012

61 Claessans et al., 2011, p. 19

62 Fung et al., 2004, p. 14

63 véase también Seidman \& Jakabovics, 2009; Bacon, 2009; Englund \& Vihriala, 2008; Bergstrom et al., 2003

64 Claessans et al., 2011

65 Gotham, 2006

66 Byrne, 2016

67 McKinsey, 2009 
mercado en general. El ejemplo más famoso aquí es la creación por parte de la RTC de los bonos de titulización hipotecaria sobre inmuebles comerciales (commercial mortgage-backed securities: CMBS), analizados en el trabajo pionero de Kevin Fox Gotham $^{68}$. La SAREB en España ha creado un mecanismo llamado Fondos de Activos Bancarios (FAB), que son paquetes de activos que se venden como Empresa Conjunta y que tienen ventajas fiscales específicas, especialmente para inversores no nacionales. Se diseñan para venderse a inversores institucionales y, según la SA$\mathrm{REB}^{69}$, se inspiran en la titulización.

\section{La globalización del sector inmobiliario}

El caso de la RTC en EEUU y la experiencia europea reciente tras la CFG indican que las SGA, al responder a las crisis de iliquidez, pueden intensificar la integración del sector inmobiliario local con los flujos globales de capital. Gotham sostiene que la creación de los CMBS por parte de la RTC jugó ese papel, principalmente al disociar los flujos de ingresos asociados a inmuebles comerciales de los edificios concretos arraigados en lugares específicos. Al juntar flujos de ingresos y desvincularlos de las condiciones locales, el proceso de titulización convierte los activos inmobiliarios en inversiones atractivas a nivel global. La RTC fue impulsada por la ausencia de compradores para sus activos, y la consecuente necesidad de conseguir nuevos inversores. La NAMA y la SAREB han sido impulsadas por dinámicas muy parecidas. Ambas sociedades han buscado atraer a entidades financieras y empresas inmobiliarias internacionales a sus mercados internos ${ }^{70}$. Lo han hecho mediante las estrategias de gestión de activos ya resumidas. En el caso de la NAMA, la creación de carteras de activos con un valor de más de 100 millones de euros se ha diseñado para atraer a grandes entidades financieras, como las empresas de capital riesgo y los fondos de cobertura. Además, la provisión de financiación de las ventas y la creación de Empresas Conjuntas por parte de la NAMA también se dirigen a estos actores; el banco malo irlandés ha utilizado estas medidas para trabajar con gigantes internacionales como Oaktree Capital (fondo de cobertura ubicado en Los Ángeles) y Lone Star Capital (empresa de capital riesgo ubicada en Texas). De manera similar, y como se ha señalado anteriormente, los FAB de la SAREB se han diseñado específicamente para atraer a inversores internacionales. Es importante destacar que un aspecto clave de todas estas estrategias es que consiguen una escala muy importante en cuanto al enorme volumen de activos que son capaces de juntar. Las SGA se hallan en una situación única para hacer esto tanto por el gran volumen de activos que adquieren como por el período corto de tiempo en el que buscan enajenar sus activos.

Examinar el mercado europeo actual en relación con la venta de activos devaluados y los préstamos inmobiliarios comerciales en general proporciona más pruebas convincentes de la interacción entre las SGA y las entidades financieras in-

68 Gotham, 2006; véase también Ashton, 2011

69 SAREB, 2014

70 Byrne, 2016 
ternacionales en la configuración de mercados inmobiliarios financiarizados tras la CFG. En un informe reciente, Cushman \& Wakefield ${ }^{71}$ estiman que las SGA europeas tienen casi 264 mil millones de euros en activos inmobiliarios devaluados y son actualmente los vendedores más activos del mercado. Están vendiendo mayoritariamente a empresas de capital riesgo en EEUU. Los compradores más significativos de los activos de las SGA europeas entre 2012 y 2014 son los siguientes: Lone Star (33\%); Cerberus (17\%); Carval (10\%); Deutsche Bank (7\%); JP Morgan (6\%); Otros $(27 \%)^{72}$.

Aunque quizá sea demasiado pronto para opinar sobre las consecuencias de estas dinámicas a largo plazo, la experiencia de la RTC parece indicar que las SGA pueden intensificar el vínculo entre el sector inmobiliario local y los flujos globales de capital a la larga, ampliando y expandiendo así las dinámicas asociadas a la financiarización de la ciudad ${ }^{73}$. En este sentido, la globalización del sector inmobiliario llevado a cabo por las SGA en un intento de responder a las crisis locales de iliquidez puede agravar aún más las mismas dinámicas que dieron lugar a esas crisis.

\section{La disputa contra los bancos malos}

Dado lo anterior, no es ninguna sorpresa que los bancos malos representan unas instituciones altamente controvertidas que son a menudo sometidas a una importante contestación política desde la sociedad civil y los movimientos sociales. Debido al papel intensivo que juegan en reactivar los mercados inmobiliarios, las SGA reproducen, e intensifican aún más, la integración del sector inmobiliario y el financiero. Por lo tanto, intensifican la tensión entre el valor de uso y el valor de cambio de los inmuebles como «activo cuasi-financiero» ${ }^{74}$. Además, por ser sociedades públicas, politizan esta tensión ${ }^{75}$. Por ende, la disputa contra las SGA se ha enfocado fundamentalmente en el acceso a y el control sobre los activos urbanos que adquieren.

El caso más destacado en este sentido es la SAREB en España. La Plataforma de Afectados por la Hipoteca (PAH), un movimiento social de personas que enfrentan el desahucio por atrasos en el pago de la hipoteca, ha ocupado hasta 13 bloques de pisos en manos de la SAREB a lo largo del estado español ${ }^{76}$. Sostienen que, al ser la SGA española pública, también lo son los activos residenciales que tiene y que, por ende, estos activos se deben convertir en vivienda social. En Irlanda, la NAMA ha enfrentado una oposición similar. Como se ha indicado anteriormente, la NAMA adquirió principalmente inmuebles comerciales, y por eso el debate se ha centrado en la conversión de los edificios de la NAMA en usos sociales y culturales. El grupo Unlock NAMA, por ejemplo, ocupó un gran bloque de oficinas en el centro de Dublín a principios de 2012, exigiendo un uso público para los activos de la NAMA, una

71 Cushman \& Wakefield, 2014

72 Cushman \& Wakefield, 2014

73 Gotham, 2006; Ashton, 2011

74 Coakley, 1997

75 Davison, 2008

76 García-Lamarca, 2017; Colau \& Almeny, 2014 
acción que alimentó un debate más amplio en la sociedad civil y en los movimientos sociales irlandeses sobre la importancia de extraer un beneficio social del banco malo ${ }^{77}$. Además, políticos y movimientos sociales han cuestionado duramente la venta de los activos de la NAMA a «fondos buitres» en el contexto de una crisis habitacional que sigue en curso. Por ejemplo, el grupo activista Dublin Tenants Association ha protestado contra la IRES, una sociedad de inversión inmobiliaria establecida en 2014 que velozmente se ha convertido en el propietario más grande de Irlanda y que ha adquirido la mayoría de su cartera inmobiliaria de la NAMA.

En ambos casos, al politizar la tensión entre la financiarización del sector inmobiliario y el valor de uso de los inmuebles, las SGA resaltan y cristalizan el antagonismo entre las finanzas y la reproducción social, que muchos entienden como un elemento clave en la política urbana contemporánea ${ }^{78}$. García-Lamarca ${ }^{79}$ conceptualiza esto como una forma de «insurgencia urbana» que representa una práctica política relacionada con el proceso más amplio de la financiarización de la ciudad.

\section{Conclusiones}

Este artículo sostiene que las SGA salvan a los bancos al intervenir en el punto de intersección entre las finanzas y el sector inmobiliario, que parece ser un nexo clave para el sistema financiero en general. El mismo término "activo deteriorado» en cierto sentido denota la disociación de las finanzas y los inmuebles subyacentes, y como las SGA trabajan con activos deteriorados, también trabajan para restablecer este vínculo y reactivar el "círculo virtuoso" entre el valor de los inmuebles y el mundo del crédito. Resolver las crisis financieras, en este sentido muy concreto, supone trabajar sobre el espacio urbano o, para utilizar el léxico financiero, "gestionar activos». En lo anteriormente expuesto, hemos visto que esto ocurre fundamentalmente de dos maneras. Primero, las SGA siguen tratando a los inmuebles como activos financieros y pueden apropiarse directamente de ingresos que provengan de los inmuebles para el sistema financiero, a menudo haciendo uso de sus poderes legales adicionales para hacerlo. Segundo, trabajan para devolver la liquidez a los mercados inmobiliarios financiarizados en general, estabilizando precios, generando transacciones y reconectando el sector inmobiliario con flujos de capital (a menudo globales). En este sentido, este artículo sostiene que las SGA operan como lo que Moreno $^{80}$ llama un «aparato de acumulación financiera», una forma político-institucional concreta y material que trabaja en y a través del espacio urbano de una manera que sustenta el proceso de financiarización.

Si las SGA se pueden conceptualizar como un aparato de acumulación financiera, entonces cabe destacar de nuevo que son instituciones del estado y responden a las crisis financieras. El análisis expuesto aquí y el caso de las SGA parece indicar que

77 O'Broin, 2012; Provisional University, 2012

78 Fields, 2014; Observatorio Metropolitano, 2012; Provisional University, 2012

79 García-Lamarca, 2017

80 Moreno, 2014 
las crisis ofrecen el contexto en el cual nuevas formas político-institucionales impulsadas por el estado surgen para posibilitar la financiarización, o quizá la re-financiarización, de la ciudad. En este sentido, utilizo y amplio el campo de estudios de Philip Ashton y Kevin Fox Gotham sobre la experiencia del «banco malo» en EEUU. Ambos autores sostienen que la RTC es importante en el sentido de que mientras intentaba responder a la crisis de ahorros y préstamos y lidiar con los activos deteriorados, su creación de los CMBS dio luz tanto a un nuevo mercado rentable como a un nuevo mecanismo para conectar el sector inmobiliario local con flujos globales de capital ${ }^{81}$. Gotham sostiene que lo que está en juego aquí no es simplemente la globalización de la inversión inmobiliaria, sino más específicamente el papel activo del estado en fabricar los circuitos mediante los cuales esto puede ocurrir. Para Ashton $^{82}$, es destacable que la RTC fue establecida para responder a condiciones de crisis, ya que éstas actúan como «momentos productivos» en el proceso de financiarización en el sentido de que las respuestas estatales pueden generar nuevas formas de intervención gubernamental y hasta nuevos mercados, fuentes de beneficios e instrumentos financieros, y todos ellos sobreviven al momento de crisis. Las crisis financieras, por tanto, ponen en juego nuevos poderes estatales, desplegados en un momento de pánico, y esta dinámica se ha «extendido dramáticamente» desde los años setenta ${ }^{83}$. El análisis expuesto aquí apoya estas aportaciones y propone que las crisis financieras y la respuesta estatal a ellas son momentos claves para investigar la relación entre el estado, las finanzas y el espacio urbano.

Enfocarnos en la resolución de las crisis financieras también es importante porque llama nuestra atención sobre la importancia, a nivel conceptual y metodológico, de situar los procesos de financiarización dentro de las dinámicas cíclicas que caracterizan los mercados inmobiliarios financiarizados. Como se ha mencionado anteriormente, toda la literatura relevante está de acuerdo en que la financiarización de la ciudad es algo inherentemente propenso a las crisis ${ }^{84}$. Por lo tanto, y especialmente considerando el caos de los últimos años, hay un conjunto importante de investigaciones sobre las burbujas financiero-inmobiliarias y cómo resultan en

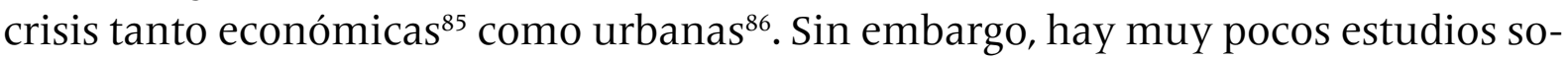
bre cómo tales crisis se resuelven y sobre la economía política urbana del proceso de resolución. En este sentido, muchas veces se presta insuficiente atención al carácter cíclico de los mercados inmobiliarios financiarizados, es decir, cómo atraviesan períodos de auge y caída, y el papel de las instituciones estatales en ello.

Por último, y como ya se ha mencionado, todo esto tiene una clara dimensión política. Las SGA son una forma de intervención pública orientada hacia el rescate de instituciones privadas con ánimo de lucro. Han dado lugar a numerosos debates

81 Ashton, 2011; Gotham, 2006

82 Ashton, 2011

83 Ashton, 2011, 1802

84 López \& Rodríguez, 2010

85 Brenner, 2006; Lapavitsas, 2013; O'Riain, 2012, 2014

86 Aalbers, 2012; López \& Rodríguez, 2011; O’Callaghan et al., 2014 
en diferentes países como parte de las críticas generales de los rescates bancarios y la subordinación del estado al sistema financiero ${ }^{87}$. Los debates mediáticos a menudo se centran en los impactos fiscales y económicos de las SGA, en lugar de sus dimensiones urbanas. Sin embargo, en Irlanda y España, las SGA han sido cuestionadas bajo el argumento de que deben tener resultados más ventajosos para las ciudades y los que viven en ellas. El espacio no permite analizar este asunto en más detalle, pero el antagonismo que surge alrededor de las SGA puede ser una nueva línea de investigación interesante, planteando preguntas teóricas sobre las formas de antagonismo político que corresponden a la financiarización y sobre una política pública que pueda incorporar resultados urbanos progresistas en la legislación que crea las SGA.

\section{Bibliografía}

AALBERS, Manuel B. Subprime cities and the twin crises. In Aalbers, Manuel. Subprime cities: the political economy of mortgage markets. Chichester: WileyBlackwell, 2012. P. 3-22.

ASHTON, Philip. The financial exception and the reconfiguration of credit risk in US mortgage markets. Environment and Planning A: Economy and Space, 2011, Vol. 43(8), p. 1796-1812.

BACON, Peter. Evaluation of options for resolving property loan impairments and associated capital adequacy of Irish credit institutions: Proposal for a National Asset Management Agency (NAMA) and associated required policy Initiatives. Dublin: National Treasury Management Agency, 2009.

BERGSTROM, Clas, Englund, Peter, Thorell, Per. Securum and the Way out of the Swedish Banking Crisis. Summary of Report Commissioned by the Center for Business and Policy Studies SNS, 2003.

BESWICK, Joe, Alexandri, Georgia, Byrne, Michael, Vives-Miró, Sonia, Fields, Desiree, Hodkinson, Stuart, Janoschka, Michael. Speculating on London's housing future: The rise of global corporate landlords in 'post-crisis' urban landscapes. City, 2016, Vol. 20(2), p. 321-341.

BLACKBURN, Robin. Finance and the fourth dimension. New Left Review, 2006, Vol. 39, p. 39-70.

BRENNER, Robert. The economics of global turbulence. London: Verso, 2006.

BYRNE, Michael. Generation rent and the financialization of housing: a comparative exploration of the growth of the private rental sector in Ireland, the UK and Spain. Housing Studies, 2019 (early online version).

BYRNE, Michael. Bad banks: the urban implications of Asset Management Companies. Journal of Urban Research and Practice, 2016, Vol. 40(1), p. 31-45. 
PAZARBASIOGLUU, Ceyla, Laeven, Luc, Nedelescu. Oana M, Claessens, Stijn, Valencia, Fabian, Dobler, Marc, Seal, Katharine. Crisis management and resolution: Early lessons from the financial crisis. International Monetary Fund, 2011. https://www.imf.org/external/pubs/ft/sdn/2011/sdn1 105.pdf [Consulta 03 de febrero 2017]

CARRUTHERS, Bruce G., Arthur L. Stinchcombe. The social structure of liquidity: Flexibility, markets, and states. Theory and Society, 1999, Vol. 28(3), p. 353-382. CHSTIPHOERS, Brett. On voodoo economics: theorising relations of property, value and contemporary capitalism. Transactions of the Institute of British Geographers, 2010, Vol. 35(1), p. 94-108.

CLAESSENS, S., Pazarbasioglu, C., Laeven, L. Dobler, M., Valencia, F., Nedelescu, O. and Seal, K. Crisis management and resolution: early lessons from the financial crisis. International Monetary Fund Staff Discussion Note, 2011. URL: https:// www.imf.org/external/pubs/ft/sdn/2011/sdn1105.pdf

COAKLEY, John. The integration of property and financial markets. Environment and Planning A: Economy and Space, 1994, 26(5), p. 697-713.COLAU

COLAU, Ada, ALEMANY, Adria. Mortgaged lives: From the housing bubble to the right to housing. London: Journal of Aesthetics and Protest Press, 2014.

CUSHMAN and Wakefield. European Real Estate Loan Market Q3, 2014. http:// www.worldpropertyjournal.com/downloads/European-CRE-Loan--REO-SalesMarket-Q3- 2014.pdf [Consulta 18 de marzo de 2018].

DAVISON, Lee. Politics and policy: The creation of the Resolution Trust Corporation. FDIC Banking Review, 2008, Vol. 17(2), p. 17-43.

DEPARTMENT of Finance. National Assets Management Agency: Section 227 Review. Dublin: Stationary Office, 2017.

ENGLUND, P., Vihriälä, V. The great financial crisis in Finland and Sweden: Similar but not quite the same. In JONUNG, Lars, KIANDER, Jaakko, VARTIA, Pentti. The great financial crisis in Finland and Sweden: the Nordic experience of financial liberalization. Cheltenham: Edward Elgar Publishing, 2009.

FIELDS, Desiree. Constructing a new asset class: property-led financial accumulation after the crisis. Economic Geography, 2018, Vol. 94(2), p. 118-140.

FIELDS, Desiree. Contesting the financialization of urban space: Community organizations and the struggle to preserve affordable rental housing in New York city. Journal of Urban Affairs, 2014, Vol. 37(2), p. 144-165

FUNG, Ben, Jason George, Stefan Hohl, and Guonan Ma. Public asset management companies in East Asia. Financial Stability Institute, occasional paper No 3. Basel: Bank for International Settlements. 2004.

GARCIA-LAMARCA, Melisa. From occupying plazas to recuperating housing: Insurgent practices in Spain. International Journal of Urban and Regional Research, 2017, Vol. 41(1), p. 37-53. 
GOTHAM, Kevin Fox. The secondary circuit of capital reconsidered: Globalization and the U.S. real estate sector. American Journal of Sociology, 2006, Vol. 112(1), p. 231-75.

GUIRONNET, Antoine., \& Halbert, Ludovic. The financialization of urban development projects: Concepts, processes, and implications. Working Paper n14-04, 2014. https://hal.archives-ouvertes.fr/hal- 01097192/document [Consulta 01 de enerero de 2018].

GUITIERREZ, Aaron., \& Domènech, Antoni. Geografía de los desahucios por ejecución hipotecaria en las ciudades españolas: evidencias a partir de las viviendas propiedad de la SAREB. Revista de Geografía Norte Grande, 2017, Vol. $67,33-52$.

HARVEY, David. Limits to Capital. London: Verso, 2006.

HARVEY, David. Rebel Cities: from the right to the city to the urban revolution. London: verso, 2012.

IMMERGLUCK, Dan., \& Law, Jonathan. Investing in crisis: The methods, strategies, and expectations of investors in single-family foreclosed homes in distressed neighborhoods. Housing Policy Debate, 2014, Vol. 24(3), p. 568-593.

INGVES, Stefan N., Seelig, Steven, He, Dong. Issues in the establishment of asset management companies. Washington: International Monetary Fund, 2004.

KELLY, Sinead. Light-touch regulation: The rise and fall of the Irish banking sector. In MACLARAN, Andrew and Sinead Kelly, Neoliberal Urban Policy and the Transformation of the City: Reshaping Dublin. Basingstoke: Palgrave Macmillan, 2014, p. 37-52.

KITCHIN, Rob, O'Callaghan, Cian, Boyle, Mark, Gleeson, Justin, Keaveney, Karen. Placing neoliberalism: The rise and fall of Ireland's Celtic Tiger. Environment and Planning A: Economy and Space, 2012, Vol. 44(6), p. 1302-26.

KITZMANN, Robert. Private versus state-owned housing in Berlin: Changing provision of low-income households. Cities, 2017, 61, 1-8.

KLINGEBEIL, Daniela. (2000). The use of Asset Management Companies in the resolution of banking crises: cross-country experiences. World Bank, 2000, <http:// elibrary.worldbank.org/doi/pdf/10.1596/1813-9450-2284> [Consulta 22 marzo de 2018]

LAPAVITSAS, Costas. The financialization of capitalism: 'Profiting without producing'. City: Analysis of urban trends, culture, theory, policy, action, 2013, Vol. 17(6), p. 792-805.

LOPEZ, Isidro, Rodríguez, Emanuel. Fin de ciclo: Financiarización, territorio y sociedad de propietarios en la onda larga del capitalismo hispano. Madrid: Traficantes de Sueños, 2010.

LOPEZ, Isidro, Rodríguez, Emanuel.. The Spanish model. New Left Review, 201 1, Vol. 69 , p. 5-29. 
MACLARAN, Andrew, Kelly, Sinead. Irish neoliberalism and neoliberal urban policy. In MACLARAN, Andrew; KELLY, Sinead. Neoliberal Urban Policy and the Transformation of the City: Reshaping Dublin. Basingstoke: Palgrave Macmillan, 2014, p. 20-36.

MCCABE, Conor. Sins of the Father: Tracing the Decisions that Shaped the Irish Economy. Dublin: The History Press Ireland, 2011.

MCKINSEY. Good riddance: Excellence in managing wind-down portfolios. Working Papers on Risk, 2012.

MCKINSEY. (2009). Understanding the bad bank. McKinsey on Corporate and Investment Banking 2009, Vol. 9 (Autumn). http://www.mckinsey.com/ insights/financial_services/understanding_the_bad_bank [Consulta 01 marzo 2018]

MORENO, Luis. The urban process under financialized capitalism. City: analysis of urban trends, culture, theory, policy, action, 2014, Vol. 18(3), p. 244-268.

NAMA. NAMA Quarterly Report. Dublin: NAMA, 2012.

O'BROIN, Michael. Ireland should renege on $€ 30$ billion NAMA bonds. Village Magazine, 2012, http://www.villagemagazine.ie/index.php/2012/04/ unlocknama/ [Consulta 25 abril 2015].

OBSERVATORIO Metropolitano. Crisis and Revolution in Europe. Madrid: Traficantes de Sueños, 2012.

O'CALLAGHAN, Cian, Boyle, Mark, Kitchin, Rob. Post-politics, crisis, and Ireland's 'ghost estates'. Political Geography, 2014, Vol. 42, p. 121-133.

O RIAIN, Sean. The crisis of financialisation in Ireland. The Economic and Social Review, 2012, Vol. 43(4), p. 497-533.

O'RIAIN, Sean. The rise and fall of Ireland's Celtic Tiger: liberalism, boom and bust. Cambridge: Cambridge University Press, 2014.

PROVISIONAL University.Financialization and the enclosure of the city: the right to the city and the right to housing in contemporary Ireland and Spain. http:// provisionaluniversity.files.wordpress.com/2013/07/financialization-andenclosure-pamphletsingle-space.pdf [Consulta 01 augusto 2018]

RACO, Mike., \& Henderson, Steven. Flagship regeneration in a global city: The re-making of Paddington Basin. Urban Policy and Research,2009, Vol. 27(3), p. 301-314.

REINHART, Carmen, Rogoff, Kenneth. This time is different: eight centuries of financial folly. New Jersey: Princeton University Press, 2009.

RUTLAND, Ted. The financialization of urban redevelopment. Geography Compass, 2010, Vol. 4(8), p. 1167-78.

SAREB. (2014). Dossier de Prensa de SAREB. https://www.sareb.es/en-en/pressoffice/documentation/Documents/Press\%20kit-\%20may\%202014_EN.pdf [Consulta 08 octubre 2017]. 
SEIDMAN, Ellen, Jakabovics, Andrew. Learning from the past: the asset disposition experiences of the home owners' loan corporation, the resolution trust corporation, and the asset control area program. Community Development Investment Review, 2009, Vol. 1, p. 43-52.

STIGLITZ, Joseph. A Bank Bailout That Works. The Nation, 2009, http://www. thenation.com/article/bank-bailout-works\# [Consulta 17 de Abril de 2017].

STUTTS, William, Watts, Wesley. Of Herring and sausage: Nordic responses to banking crises as examples for the United States. Texas International Law Journal, 2009, Vol. 44, p. 577-627.

VAN DER ZWAN, Natascha. (2012). State of the art: making sense of financialization. Socio-Economic Review, 2012, Vol. 12(1), p. 99-129.

WALDRON, Richard. (2018) Capitalizing on the State: The political economy of Real Estate Investment Trusts and the 'Resolution' of the crisis, Geoforum, 90, pp. 206-218.

WEBER, Rachel. Extracting value from the city: neoliberalism and urban redevelopment. Antipode, 2002, Vol. 34(3), p. 519-40.

WIJBURG, Gertjan. \& Aalbers, Manuel. B. The alternative financialization of the German housing market, Housing Studies, 2017, 32(7), p. 968-989.

WISSOKER, Peter, Fields, Desiree, Weber, Rachel, Wyly, Elvin. Rethinking real estate finance in the wake of a boom: a celebration of the twentieth anniversary of the publication of the double issue on property and finance. Environment and Planning A'. Environment and Planning A: Economy and Space, 2014, Vol. 46(12), p. 2787-2794.

(C) Copyright: Michael Byrne, 2020

(c) Copyright: Scripta Nova, 2020.

Ficha bibliográfica:

BYRNE, Michael. Los «bancos malos» y la resolución de la crisis financiero-inmobiliaria en Europa. Scripta Nova. Revista Electrónica de Geografía y Ciencias Sociales. Barcelona: Universidad de Barcelona, 1 de Septiembre de 2020, vol. XXIV, nº 645. [ISSN: 1138-9788] 